ACTA UNIVERSITATIS LODZIENSIS

Folia Litteraria Romanica 16, 2021

https://doi.org/10.18778/1505-9065.16.19

Radka Mudrochová

Université Charles de Prague

(iD) https://orcid.org/0000-0002-8718-6922

radka.mudrochova@ff.cuni.cz

Nyeberth Emanuel Pereira dos Santos

Universidade Federal de Campina Grande

(iD https://orcid.org/0000-0002-6037-1108

nyeberth@gmail.com

\title{
Mon lapin, ma biche ou loulou? La fréquence d'emploi des surnoms d'amour pour femmes et pour hommes en français de France et en français québécois
}

\begin{abstract}
RÉSUMÉ
L'objectif de cette communication est d'analyser la productivité et l'usage des surnoms d'amour pour femmes et pour hommes dans le contexte francophone, notamment en France et au Québec. À partir des dispositifs lexicographiques et des ressources de la Toile, nous avons élaboré un corpus de mots que nous analysons plus en détail avec des outils de la linguistique de corpus, à savoir sur la plateforme Néoveille et dans les corpus slovaques Aranea permettant de mesurer les fréquences d'emploi dans les deux contextes distincts français et québécois. Premièrement, nous allons caractériser et classifier, en général, les surnoms affectifs pour femmes et pour hommes. Deuxièmement, nous allons présenter les mots recueillis pour notre analyse et nous allons les commenter en fonction des processus de leur formation ou de leur appartenance à un domaine déterminé. Finalement, nous allons vérifier la fréquence d'emploi et la productivité d'un échantillon de mots choisi dans des corpus linguistiques choisis, et nous allons comparer les deux contextes de notre recherche pour pouvoir nous prononcer sur leurs ressemblances ou différences.
\end{abstract}

MOTS-CLÉS - surnoms d'amour, diminutifs, langue française, hypocoristiques, corpus Aranea

\section{Mon lapin, ma biche or loulou? The Frequency of Use of Love Nicknames for Women and Men in French in France and in Québécois French}

\section{SUMMARY}

The objective of this article is to analyze the use of love nicknames for women and men in the French-speaking context, especially in France and Quebec. From the lexicographic devices and 
resources of the Web, we have developed a corpus of words which we have analyzed in more detail with the tools of corpus linguistics, namely on the platform Néoveille and in the corpora Araneum allowing to measure the frequencies of employment in the two distinct contexts, French (from France) and Québécois French. This article has a threefold purpose. First, we will try to characterize and classify the affectionate nicknames. Second, we will present all of the words collected for our analysis and we will comment on them according to the processes of their formation or their belonging to a specified domain. Finally, we will verify the frequency of use of a sample of words chosen from the linguistic corpus mentioned above and we will notably compare the two contexts of our research to be able to decide on their similarities or differences.

KEYWORDS - love nicknames, diminutives, French language, hypocoristicon, Aranea corpora

\section{Introduction}

L'objectif de cet article ${ }^{1}$ est d'analyser la fréquence d'emploi et l'usage des surnoms d'amour pour femmes et pour hommes dans le contexte francophone, notamment en France et au Québec.

À partir des dispositifs lexicographiques et des ressources de la Toile, nous avons élaboré un corpus de mots que nous avons analysé plus en détail avec des outils de la linguistique de corpus, à savoir sur la plateforme Néoveille et dans les corpus slovaques Aranea permettant de mesurer les fréquences d'emploi dans les deux contextes distincts français (de France) et québécois.

En effet, le présent article a un triple objectif. Premièrement, nous allons essayer de caractériser et de classifier les surnoms d'amour au sein de la linguistique. Deuxièmement, nous allons présenter l'ensemble des mots recueillis pour notre analyse et nous allons les commenter selon les procédés de leur formation ou de leur appartenance à un domaine spécifié. Finalement, nous allons vérifier la fréquence d'emploi d'un échantillon de mots choisis dans les corpus linguistiques cités supra et nous allons notamment comparer les deux contextes de notre recherche pour pouvoir nous prononcer sur leurs ressemblances ou leurs différences.

\section{Le surnom d'amour en tant que notion linguistique}

Les surnoms d'amour appartiennent en linguistique à plusieurs catégories, ils font partie des diminutifs, des expressions hypocoristiques ou des mots à valeur méliorative. Les linguistes Nicole et Jean Tournier (2009 : 178-179)

\footnotetext{
${ }^{1}$ Le présent article s'inscrit dans le Projet Européen du Développement Régional " Créativité et adaptabilité comme conditions du succès de l'Europe dans un monde interconnecté » (No. CZ.02 .1.01/0.0/0.0/16_019/0000734) et a bénéficié du soutien du projet de l’Université Charles « Progres Q10 : Le langage dans les changements de temps, d'espace et de culture ».
} 
définissent un hypocoristique comme : " une lexie exprimant une intention affectueuse, y compris des noms d'animaux précédés du possessif $\mathrm{ma} / \mathrm{mon}$, adressés à des personnes : ma poule, ma cocotte, mon poussin, mon poulet, ma caille, ma biche, ma puce, mon chat, mon lapin ». En revanche, le Trésor de la langue française informatisé (TLFi, en ligne) reprend la définition de Mounin (1974: 166) et lie les hypocoristiques au langage des enfants ou à ses imitations en distinguant quatre procédés de création de ces lexies, à savoir : (1) le redoublement (fifille), (2) les suffixes, dits diminutifs (fillette), (3) l'abrègement (Mado), (4) le choix de termes conventionnellement hypocoristiques (mon petit poulet, mon chou).

Selon des spécialistes (cf. par exemple : De Carlo 2003 ; Falk 2004), l'usage des hypocoristiques ou des surnoms amoureux, se rapporte au « baby-talk» de notre enfance. En effet, les petits animaux (de compagnie) sont engagés dans le discours pour parler de son amoureux/amoureuse. Il s'agit d'une relation métaphorique avec l'univers des enfants. L'usage des diminutifs, voire des hypocoristiques, confirme ici la relation intime entre les participants (Dressler; Merlini Barbaresi, 1994 : 193).

Les diminutifs appartenant pour leur côté affectif aux hypocoristiques (cf. Tournier, 2009 : 113) peuvent prendre différentes formes et être classifiés notamment selon leur procédé de création. Ainsi, Marek Nekula, un linguiste tchèque (cité via Jandeková, 2019: 15), distingue, pour ce qui est de la diminution des hypocoristiques, d'une part les formes analytiques comprenant la création par des mots / des radicaux à valeur diminutive ou par le redoublement de mots, et d'autre part les formes synthétiques incluant les procédés tels que la suffixation / le redoublement de syllabes / l'abréviation.

Étant donné que le français est une langue analytique, la suffixation n'est pas le moyen le plus fréquent dans la formation des diminutifs, contrairement à la langue tchèque. La stagnation de la création synthétique est donnée, selon Šabršula (1983 : 82-83), par l'augmentation de la fréquence de l'opération diminutive à l'aide de l'adjectif petit dont la fréquence est plus élevée en comparaison avec les équivalents dans les autres langues romanes. Pour ce qui est du redoublement, également productif en français, il comprend deux procédés distincts : (1) le redoublement de la consonne et de la voyelle initiales propre à certains prénoms $(\mathrm{Fi}$ fine); (2) le redoublement de toute une unité lexicale indépendante (chouchou). Ces derniers exemples cités appartiennent notamment au langage enfantin accompagné d'intentions hypocoristiques (Loulou, Mimi, Zizi) qui « [...] répondent au besoin ludique humain $[\ldots]$ » et «[...] détiennent une expressivité et donc une communication précise » (Aitokhuehi, $1996: 9$ ).

Le dernier procédé de la formation synthétique évoquée par Nekula recouvre l'abréviation, notamment sa sous-catégorie la troncation (cf. Fridrichová, 2014 : 37-38), qui par l'enlèvement de certains phonèmes permet de créer des prénoms à valeur diminutive (Théo $=$ Théophile, Mag'= Magali, Steph/Stef $=$ Stéphane, 
Nico $=$ Nicolas $)$. Grevisse $(2000: 252)$ ajoute à ce propos les altérations variées des prénoms $($ Popol $=$ Paul, Mimile $=$ Émile, Marilou $=$ Marie-Louise $)$ incluant d'habitude plusieurs procédés de formation des mots.

\section{Le surnom d'amour en tant que notion non-linguistique}

La classification des surnoms d'amour par procédé de formation ne représente pas leur unique distinction. Il est également possible de les classer par domaine auquel appartient le mot clé du surnom. D'où, par exemple, les surnoms ${ }^{2}$ :

- gourmands (mon sucre d'orge, mon bonbon, ma truffe, mon chou)

- précieux (mon trésor, mon bijou, ma perle rare)

- enfantins (mon bébé, mon doudou)

- classiques (mon cour, mon amour, mon chéri)

- vieillots (papa, maman)

- anglicismes (baby, darling, sugar, beauty)

- autres langues étrangères, p. ex. : arabe : habibi 'mon amour', azizi 'mon chéri', galbi 'mon cœur', espagnol : mi vida 'ma vie', media naranja 'ma moitié', cariño 'mon trésor', mi bichito 'mon petit insecte', italien : dolce metà 'ma moitié', mio caro 'mon chéri', mio pulcino 'mon poussin', mio tesoro 'mon trésor'

- romantiques (ma princesse aux petits pieds, ma sirène de miel)

- diminutifs de prénoms (mumu, titi, yoyo, lolotte, riri)

- humoristiques (ma grosse mémère, Casimir (en raison du gros ventre), Choubaka (en raison du système pileux hyperdéveloppé), Mimie Mathy (en raison de la petite taille)

- animaux (ma biche, mon chat, mon canard, ma poulette, ma tourterelle).

Certaines catégories citées ci-dessus trouvent sur Internet d'autres dénominations, par exemple, les anglicismes tels que baby, darling, sugar, beauty sont aussi nommés surnoms show-biz ${ }^{3}$ et les surnoms enfantins sont appelés régressifs. Évidemment, parfois, le même surnom peut appartenir à plusieurs domaines comme c'est le cas des appellations pupuce ou chaton (surnoms enfantins/animaux) ou de la catégorie des surnoms de bêtes sauvages sensuels (mon grand buffle, mon tigre du Bengale, ma tigresse, mon grand loup des Steppes) faisant partie des surnoms animaux en général.

${ }^{2}$ Repris des sites : https://pressfrom.info/fr/lifestyle/vie-perso/-20544-que-signifient-vos-surnomsamoureux.html, https://mesclesdubonheur.com/surnoms-amoureux-quels-sont-les-votres/, https:// www.journaldesfemmes.fr/couple/conseils-amoureux/1325568-surnom-amoureux-idees-signification/, http://www.avenue-romantique.fr/2015/07/200-surnoms-amoureux-pour-homme-etfemme.html, consultés le 10 septembre 2019.

${ }^{3} \mathrm{https}$ //www.parents.fr/etre-parent/couple/psycho-couple/que-signifient-vos-surnoms-amoureux-221, consulté le 23 novembre 2019. 


\section{Les surnoms d'amour selon leur popularité d'usage sur la Toile}

Avant de présenter notre corpus de recherche ainsi que les résultats de nos analyses, nous aimerions attirer, dans un premier temps, l'attention sur l'enquête de la marque Mon chéri réalisée en 2019 auprès de 175000 personnes $^{4}$, pour la fête de la Saint-Valentin et dont l'objectif était de recueillir, grâce à un sondage disponible en ligne, les surnoms amoureux préférés des Français. Le résultat global de ce sondage, présentant les cinq surnoms amoureux des Français, est illustré par le graphique 1 .

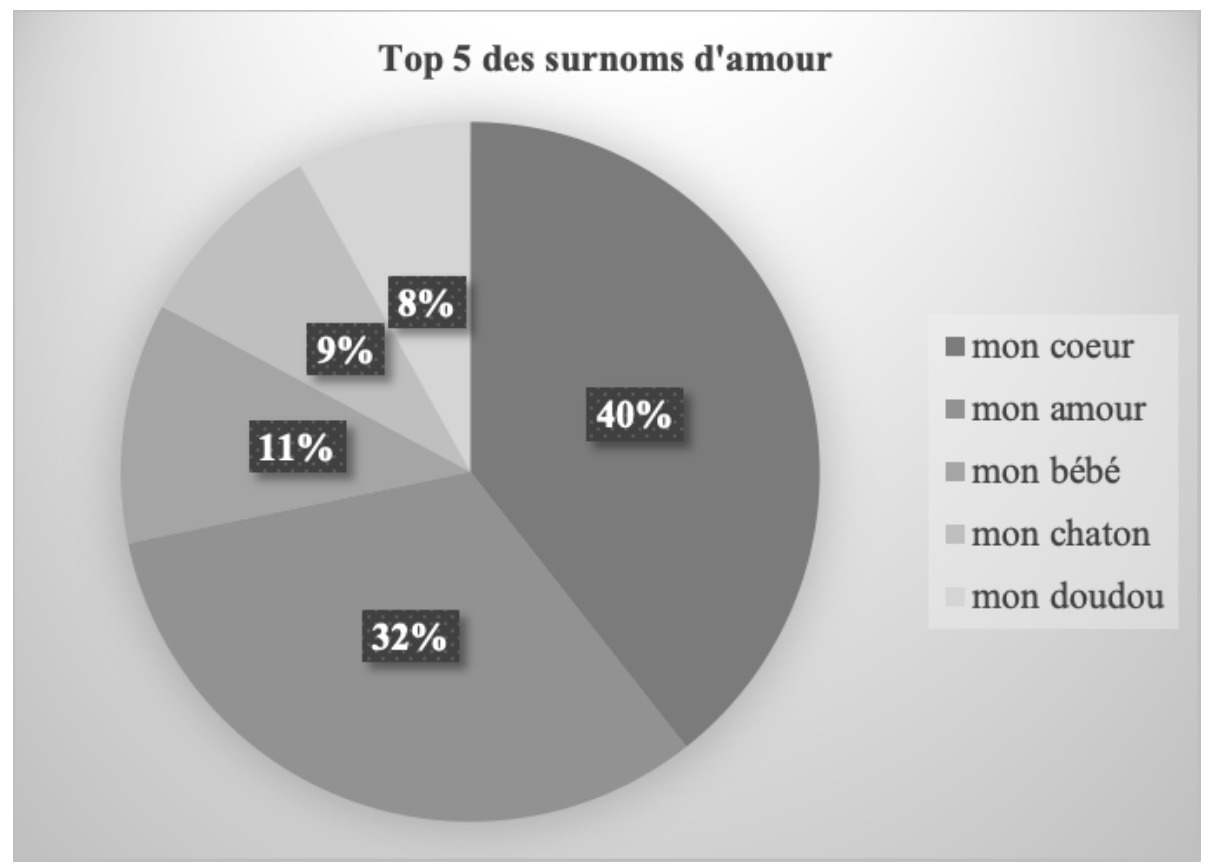

Graphique 1. Les cinq surnoms d'amour préférés des Français selon l'enquête Mon chéri

Le graphique montre que le surnom préféré des Français est mon cœur, cité par $40 \%$ des personnes ayant répondu, suivi de mon amour avec $32 \%$. Les trois autres surnoms affichent des pourcentages très proches : mon bébé $(11 \%)$, mon chaton (9\%), mon doudou ( $8 \%)$.

Ce sondage nous a servi de point de départ pour nos analyses ultérieures et nous a notamment permis de constituer, dans un deuxième temps, notre propre corpus de surnoms d'amour qui sont le plus fréquemment indiqués comme « top »

${ }^{4}$ Les résultats du sondage ont été repris du site : https://www.elle.fr/Love-Sexe/Mon-mec-et-moi/Voici-le-top-5-des-surnoms-amoureux-preferes-des-Francais-3753240, consulté le 23 novembre 2019. 
ou populaires sur Internet. Leur choix provient de différents blogs Internet ${ }^{5}$ et nous avons également pris en considération l'enquête de Mon chéri. Par la suite, nous avons vérifié la présence de ces lexies dans les dictionnaires de langue générale (Petit Robert (PR), Larousse, Dictionnaire de l'Académie française (DAF), $\left.U_{\text {sito }}\right)^{6}$. La recherche a été limitée à la valeur d'affection et/ou hypocoristique. En revanche, nous avons exclu l'indication des termes d'amitié. Les résultats de cette analyse sont résumés par le tableau 1.

Tableau 1. Surnoms d'amour les plus fréquents sur Internet et leur présence dans les dictionnaires de langue générale choisis

\begin{tabular}{|l|c|c|c|c|}
\hline \multicolumn{1}{|c|}{ Lexie } & $\mathrm{PR}$ & Larousse & $\mathrm{DAF}$ & Usito \\
\hline mon cour & $\mathrm{O}$ & $\mathrm{O}$ & $\mathrm{O}$ & $\mathrm{O}$ \\
\hline mon amour & $\mathrm{O}$ & $\mathrm{O}$ & $\mathrm{O}$ & $\mathrm{O}$ \\
\hline mon bébé & $\mathrm{N}$ & $\mathrm{N}$ & $\mathrm{N}$ & $\mathrm{N}$ \\
\hline mon chaton & $\mathrm{N}$ & $\mathrm{N}$ & $\mathrm{N}$ & $\mathrm{N}$ \\
\hline mon doudou & $\mathrm{O}$ & $!$ & $!$ & $\mathrm{N}$ \\
\hline mon lapin & $\mathrm{O}$ & $\mathrm{O}$ & $\mathrm{O}$ & $\mathrm{O}$ \\
\hline mon poussin & $\mathrm{O}$ & $\mathrm{N}$ & $\mathrm{O}$ & $\mathrm{O}$ \\
\hline mon canard & $\mathrm{O}$ & $\mathrm{N}$ & $\mathrm{O}$ & $\mathrm{N}$ \\
\hline mon bichon & $\mathrm{N}$ & $\mathrm{O}$ & $\mathrm{N}$ & $\mathrm{N}$ \\
\hline mon ange & $\mathrm{O}$ & $\mathrm{O}$ & $\mathrm{O}$ & $\mathrm{O}$ \\
\hline mon trésor & $\mathrm{O}$ & $\mathrm{O}$ & $\mathrm{N}$ & $\mathrm{O}$ \\
\hline ma poupée & $\mathrm{N}$ & $\mathrm{N}$ & $\mathrm{N}$ & $\mathrm{N}$ \\
\hline mamour & $!$ & $!$ & $\mathrm{O}$ & $\mathrm{O}$ \\
\hline mon chou/ma choute & $\mathrm{O}$ & $\mathrm{N}$ & $\mathrm{O}$ & $\mathrm{O}$ \\
\hline chérile & $\mathrm{O}$ & $\mathrm{O}$ & $\mathrm{O}$ & $\mathrm{O}$ \\
\hline chouchou-loulou & $\mathrm{N}$ & $\mathrm{N}$ & $\mathrm{N}$ & $\mathrm{O}$ \\
\hline ma puce & $\mathrm{O}$ & $\mathrm{N}$ & $\mathrm{O}$ & $\mathrm{O}$ \\
\hline TOTAL OUI & 10 & 7 & 10 & 11 \\
\hline
\end{tabular}

En observant le tableau 1, nous remarquerons que le dictionnaire Usito (dictionnaire créé au Québec) reflète le plus les valeurs d'affection. Ensuite, il est à noter que les cinq lexies mises en gris sont toutes présentes (avec la

${ }^{5}$ Repris des sites : https://sobusygirls.fr/2019/03/02/petits-surnoms-mignons-couple-amoureux/, https://www.fourchette-et-bikini.fr/sexo/top-10-des-surnoms-amoureux-les-plus-utilises-37578. html, consultés le 9 octobre 2019.

${ }^{6}$ Pour les quatre dictionnaires, nous avons utilisé les versions en ligne, consultées entre les 23-26 octobre 2019. 
mention de leur valeur hypocoristique) dans les quatre dictionnaires choisis. En revanche, il y a trois termes d'affection qui ne sont pris en considération par aucun dictionnaire, à savoir mon bébé, mon chaton, ma poupée. Dans ce contexte, il faut souligner que l'expression mon bébé représentait le troisième surnom d'amour d'après l'enquête de Mon chéri, pourtant sa signification reste absente des dictionnaires cités. Enfin, nous repérons deux particularités, marquées par des points d'exclamation dans le tableau : d'une part, cela concerne le mot mamour qui apparaît dans les dictionnaires le Petit Robert et le Larousse au pluriel, mamours, pour parler des « démonstrations de tendresse », donc le sens recherché d'un surnom d'amour est absent. D'autre part, le terme d'affection mon doudou se limite dans les trois dictionnaires (le Petit Robert, le Larousse, le Dictionnaire de l'Académie française) à un territoire francophone spécifique, les Antilles, pour désigner de manière affectueuse et familière une " femme native des Antilles » (DAF), une " jeune femme aimée (Antilles) » (PR), ou c'est une " appellation tendre donnée aux Antilles à une femme » (Larousse).

\section{Les surnoms d'amour en tant que termes d'affection dans les dictionnaires}

Dans un troisième temps, nous avons procédé à une analyse inverse en utilisant le dictionnaire le Petit Robert (la dernière version disponible en ligne) comme point de départ. Dans la recherche avancée, qui est un outil de base du dictionnaire, nous avons simplement tapé « affection » pour recueillir un ensemble de mots hypocoristiques, y compris des surnoms d'amour. Nous sommes passés au tri manuel et, au total, nous avons réuni 42 lexies/expressions qui peuvent être classifiées comme surnoms amoureux (cf. tableau 2).

Tableau 2. Surnoms d'amour en tant que termes d'affection issus du PR

\begin{tabular}{|l|l|}
\hline ma bichette & mon petit lapin en sucre \\
\hline mon biquet/ma biquette & beau/belle \\
\hline ma petite minette & chéri/-e \\
\hline choupinet/choupinette & ma douce \\
\hline chouchou/chouchoute & ma biche \\
\hline mon gros loup & mon canard \\
\hline ma petite caille & mon chat \\
\hline ma petite chatte & ma colombe \\
\hline mon petit pigeon & mon lapin \\
\hline mon petit poulet & mon loup \\
\hline
\end{tabular}


Tableau 2 (cont.)

\begin{tabular}{|l|l|}
\hline mon petit rat & mon pigeon \\
\hline mon petit coco & ma poule \\
\hline mon petit chérubin & mon poulet \\
\hline mon trésor & mon poussin \\
\hline loulou/louloute & ma puce \\
\hline ma poupoule & mon rat \\
\hline bobonne & mon cœur \\
\hline mon doudou & mon petit cœur \\
\hline mon âme & ma poulette \\
\hline ma chère âme & ma cocotte \\
\hline mon ange & mon minet \\
\hline
\end{tabular}

En respectant les catégories de classification citées supra, nous avons essayé d'analyser les surnoms issus du dictionnaire le PR plus en détail. Nous constatons que ces surnoms amoureux appartiennent majoritairement au monde animalier, soit $70 \%$ des expressions de notre corpus. Ils sont en général précédés par le déterminant possessif mon/ma (mon pigeon, ma poule, mon poulet, ma puce, etc.) et l'adjectif petit (mon petit rat, mon petit poulet), formés par dérivation (mon minet, ma bichette) ou par redoublement (loulou/louloute, ma poupoule). D'autres domaines sont plus marginaux : la flore (choupinet/-ette, chouchou/tte), le spirituel/le religieux (mon âme, ma chère âme, mon petit chérubin, mon ange), les surnoms précieux (mon trésor) et les classiques (mon cœur/mon petit cœur, mon chéri). Pour ce qui est des procédés employés pour la création des termes d'affection, $25 \%$ de l'ensemble des expressions sont accompagnées de l'adjectif petit qui, avec la dérivation (19,5\%) et le redoublement (10\%), représentent les procédés les plus productifs.

\section{La fréquence d'emploi des termes d'affection du PR en France et au Canada}

Étant donné que l'un des objectifs de cette contribution consistait dans la comparaison de l'emploi des surnoms d'amour dans deux contextes différents, celui de la France et celui du Québec, voire du Canada, nous avons procédé, dans un dernier temps, à une recherche dans les corpus de l'Académie slovaque, Aranea (cf. notamment Benko, 2018), à savoir : AFM Araneum Francogallicum Maius (FR) et AFC Araneum Francogallicum Canadiense (CA), ce qui a permis non seulement « d'exploiter de larges bases de données d'occurrences concrètes dans des contextes authentiques » comme le souligne Nádvorníková (2017 : 67), mais également de vérifier, dans notre cas, la fréquence d'usage dans les deux contextes distincts. Pour avoir un échantillon de recherche plus homogène, nous 
avons limité notre analyse au monde animalier ${ }^{7}$, représentant le domaine le plus important de toutes les catégories du PR. Les occurrences recueillies à partir de la simple requête de chaque lexie/expression dans les deux corpus linguistiques sont présentées dans le tableau 3.

Tableau 3. Fréquence d'emploi des termes d'affection (monde animalier) France vs Canada

\begin{tabular}{|l|c|c|l|c|c|}
\hline \multicolumn{1}{|c|}{ Terme d'affection } & AFM & AFC & Terme d'affection & AFM & AFC \\
\hline loulou/louloute & $4425 / 406$ & $176 / 1$ & ma bichette & 54 & 2 \\
\hline Mon biquet/ma biquette & $36 / 16$ & $0 / 0$ & ma colombe & 49 & 12 \\
\hline ma puce & 1318 & 225 & mon rat & 48 & 1 \\
\hline ma biche & 592 & 1 & mon pigeon & 18 & 2 \\
\hline ma cocotte & 174 & 37 & mon petit coco & 16 & 9 \\
\hline ma poulette & 159 & 8 & mon petit poulet & 10 & 0 \\
\hline mon minet & 149 & 0 & ma petite minette & 8 & 0 \\
\hline mon loup & 135 & 10 & mon petit rat & 5 & 0 \\
\hline ma petite chatte & 131 & 3 & ma poupoule & 3 & 0 \\
\hline mon poussin & 111 & 2 & mon gros loup & 2 & 1 \\
\hline mon canard & 55 & 2 & mon petit pigeon & 2 & 0 \\
\hline & & & ma petite caille & 1 & 0 \\
\hline
\end{tabular}

Dans le tableau 3, nous avons marqué en gris foncé les chiffres les plus élevés pour la France : loulou/louloute, ma puce, ma biche et ma cocotte, et en gris clair pour le Canada : loulou, ma puce, ma cocotte, ma colombe. Parmi ces expressions, il y en a deux, ma puce, ma cocotte, qui sont fréquemment employées dans les deux corpus. En revanche, les mots en gras soulignent les résultats marginaux pour le Canada par rapport à la France. Il s'agit notamment de la forme féminine de l'appellation loulou, donc louloute, comptant dans le corpus canadien une seule occurrence, tout comme ma biche, qui pourtant possède dans le corpus français de France 592 occ. Ensuite, nous y classons les expressions : mon biquet / ma biquette et mon minet, complètement absentes dans le corpus AFC en comparaison avec le corpus AFM.

Pour terminer cette recherche de fréquence, nous avons tapé les deux expressions ayant les chiffres les plus élevés dans les deux corpus Aranea, loulou et ma puce, sur la plateforme Néoveille, un outil linguistique mis en place en 2015 qui télécharge grâce aux canaux RSS des informations d'une soixantaine de périodiques français/francophones (cf. notamment Cartier, 2016). Même

${ }^{7}$ Nous avons exclu de notre analyse quatre termes d'affection : mon chat, mon poulet, ma poule, mon lapin, car ils affichaient trop de redondance dans les corpus étant donné qu'ils peuvent être employés dans des contextes très généraux. 
si l'objectif de la plateforme Néoveille est a priori de suivre et de repérer des néologismes, avec son moteur de recherche interne, on peut cependant consulter n'importe quel mot qui y est enregistré et visualiser ses courbes de vie, notamment son évolution temporelle, la répartition de ses occurrences par domaine, par périodique et par territoire francophone. La figure 1 montre la répartition des occurrences par territoire des deux surnoms d'amour, loulou et ma puce, sur Néoveille (recherche effectuée le 30 octobre 2019).
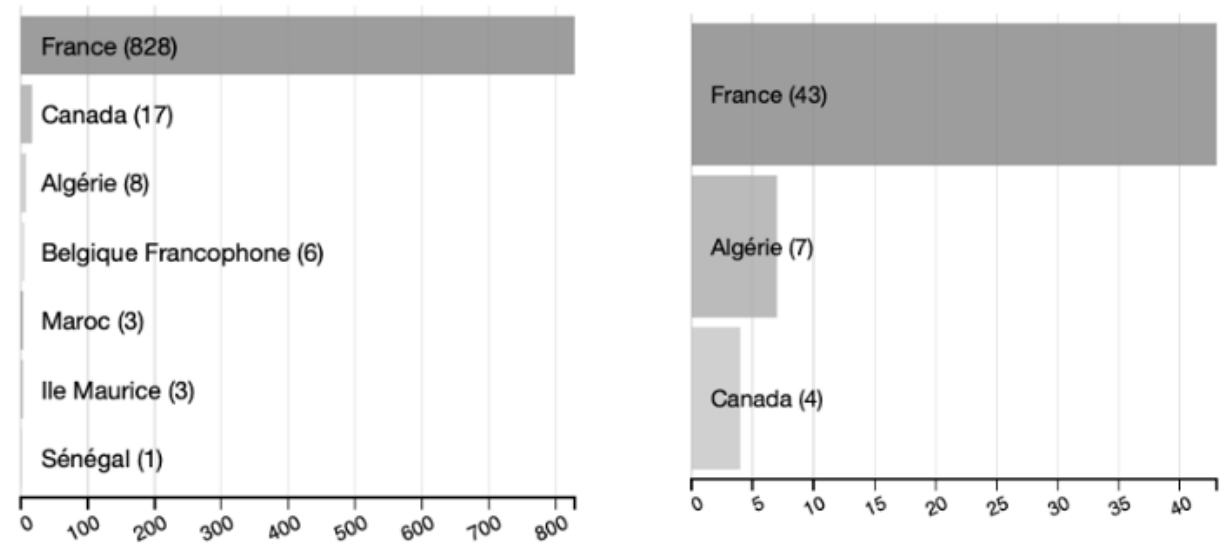

Figure 1. Répartition des occurrences des termes d'affection loulou (à gauche) et ma puce (à droite) sur Néoveille

La figure 1 révèle que le surnom loulou englobe sur Néoveille plus de territoires francophones, avec des occurrences (bien que marginales par rapport à la France) au Canada, en Algérie, en Belgique, au Maroc, etc. En revanche, l'expression ma puce est proportionnellement «moins francophone » et dans son ensemble aussi moins fréquente sur la plateforme en question.

\section{Conclusion}

Cette étude nous a permis d'observer, d'une manière générale, l'emploi des surnoms d'amour de plusieurs points de vue.

D'abord, nous remarquons que les surnoms d'amour ne possèdent pas de chapitre spécifique dans la linguistique, ils sont d'habitude classés parmi les diminutifs, les hypocoristiques ou les corpus métalinguistiques, comme dans le Petit Robert qui les désignent comme des termes d'affection.

Suite à plusieurs analyses distinctes des surnoms d'amour, nous avons découvert que : mon amour, mon cœur représentaient les appellations amoureuses 
préférées des Français, incluses dans les bases des dictionnaires de langue générale et indiquées majoritairement par les internautes. En revanche, une analyse de ces expressions, dites de préférence, a montré qu'il n'y avait aucune mention des surnoms : ma poupée, mon chaton, mon bébé dans les corpus métalinguistiques généraux. Pourtant ils étaient cités dans des palmarès de surnoms d'amour sur plusieurs sites Internet.

La recherche des termes d'affection dans le dictionnaire le Petit Robert a révélé une grande présence de mots liés au monde animalier, soit $70 \%$ de notre corpus, ainsi que d'expressions construites à partir de la formation analytique de l'adjectif petit ( $25 \%$ ). Le monde animalier représentait également le dernier point de notre recherche dans des corpus linguistiques mesurant notamment la fréquence d'emploi des lexies choisies en France par rapport au français canadien. D'une part, nous trouvons des ressemblances dans l'emploi des surnoms d'amour en France et au Canada (loulou, ma puce), et d'autre part, il y a des occurrences (complètement) absentes dans le corpus canadien (louloute, mon biquet / ma biquette, ma biche, mon minet). En outre, certaines de ces expressions sont aussi répandues dans toute la francophonie, notamment loulou.

Pour terminer, nous aimerions souligner que l'objectif de cette étude ne consistait pas en une analyse détaillée des surnoms d'amour en français de France et au Canada, mais nous voulions plutôt montrer certaines de leurs caractéristiques qui pourraient être développées dans des recherches ultérieures.

\section{Bibliographie}

AITOKHUEHI, Emmanuel (1996), "Le redoublement : expressivité et créativité lexicale », Initial(e)s, vol. 15, p. 3-11. (https://ojs.library.dal.ca/initiales/article/viewFile/5133/4637, consulté le 28 août 2019)

BENKO, Vladimír (2018), "Aranea: A Family of Comparable Gigaword Web Corpora », Web Corpora \& Corpus Linguistics Portal, Bratislava, Slovak Academy of Scientces L. Štúr Institute of Linguistics. (http://aranea.juls.savba.sk/aranea_about/index.html, consulté le 21 janvier 2020)

CARTIER, Emmanuel (2016), « Neoveille, système de repérage et de suivi des néologismes en sept langues », Neologica, $\mathrm{n}^{\circ} 10$, p. 101-131

DE CARLO, Maddalena (2003), «Affectivité et acquisition du langage », Ela. Études de linguistique appliquée, $\mathrm{n}^{\circ} 131$, p. 275-290

DRESSLER, Wolfgang Ulrich, MERLINI BARBARESI, Lavinia (1994), Morphopragmatics. Diminutives and Intensifiers in Italian, German and Other Languages, Berlin, De Gruyter

FALK, Dean (2004), « Baby talk key to evolution », The Guardian. (https://www.theguardian.com/ science/2004/dec/20/evolution.science, consulté le 21 janvier 2020)

FRIDRICHOVÁ, Radka (2014), « À propos des troncations de noms propres - les spécificités des mots onomastiques tronqués », Linguistica Pragensia, $\mathrm{n}^{\circ}$ 1, p. 34-44

GREVISSE, Maurice, GOOSSE, André (2000), Le Bon usage, Paris, Duculot

JANDEKOVÁ, Kateřina (2019), L'emploi pragmatique de diminutifs en français, mémoire de Master, Prague, Faculté des Lettres, Université Charles de Prague 
MOUNIN, Georges (1974), Dictionnaire de la linguistique, Paris, PUF

NÁDVORNÍKOVÁ, Olga (2017), « Le corpus multilingue InterCorp : nouveaux paradigmes de recherche en linguistique contrastive et en traductologie », Studii de lingvistică, no 7, p. 67-88

NEKULA, Marek (2010), « Deminutiva a augmentativa v češtině z typologického pohledu », Karlikk a továrna na lingvistiku, Brno, Host, p. 304-315

ŠABRŠULA, Jan (1983), Základy francouzské lexikologie, Praha, Státní pedagogické nakladatelství

TOURNIER, Nicole, TOURNIER, Jean (2009), Dictionnaire de lexicologie française, Paris, Ellipses

\section{Sitographie}

http://atilf.atilf.fr, consulté le 21 janvier 2020

http://unesco.uniba.sk, consulté le 28 février 2020

http://www.avenue-romantique.fr/2015/07/200-surnoms-amoureux-pour-homme-et-femme.html, consulté le 10 septembre 2019

https://fr.wiktionary.org/, consulté le 21 janvier 2020

https://larousse.fr/, consulté le 23 janvier 2020

https://lipn.univ-paris13.fr/neoveille/html/login.php?action=login, consulté le 11 janvier 2020

https://mesclesdubonheur.com/surnoms-amoureux-quels-sont-les-votres/, consulté le 10 septembre 2019

https://pr.bvdep.com/robert.asp, consulté le 23 décembre 2019

https://pressfrom.info/fr/lifestyle/vie-perso/-20544-que-signifient-vos-surnoms-amoureux.html, consulté le 10 septembre 2019

https://sobusygirls.fr/2019/03/02/petits-surnoms-mignons-couple-amoureux/, consulté le 09 octobre 2019

https://www.dictionnaire-academie.fr, consulté le 23 janvier 2020

https://www.elle.fr/Love-Sexe/Mon-mec-et-moi/Voici-le-top-5-des-surnoms-amoureux-preferesdes-Francais-3753240, consulté le 23 novembre 2019

https://www.fourchette-et-bikini.fr/sexo/top-10-des-surnoms-amoureux-les-plus-utilises-37578. html, consulté le 09 octobre 2019

https://www.journaldesfemmes.fr/couple/conseils-amoureux/1325568-surnom-amoureux-ideessignification/, consulté le 10 septembre 2019

https://www.parents.fr/etre-parent/couple/psycho-couple/que-signifient-vos-surnomsamoureux-221, consulté le 23 novembre 2019

Radka Mudrochová - docteure en linguistique, elle occupe le poste de maîtresse de conférences en linguistique française à l'Université Charles de Prague et à l'Université de Bohême de l'Ouest de Plzeň (Tchéquie). Ses recherches se centrent sur la lexicologie contrastive du français et du tchèque et sur la néologie. Récemment, elle a publié L'emprunt en question(s) (avec Alicja Kacprzak et Jean-François Sablayrolles) et plusieurs articles portant notamment sur les emprunts à l'anglais en français et en tchèque.

Nyeberth Emanuel Pereira dos Santos - doctorant en Lettres (langue et littérature françaises) à l'Université Clermont Auvergne, il occupe le poste de professeur de langue et littérature française à l'Université Fédérale de Campina Grande (UFCG), au Brésil. Ses recherches se centrent sur la littérature, la critique psychanalytique et l'enseignement du FLE. 